\title{
A BILINEAR FORM FOR SPIN MANIFOLDS
}

\author{
PETER S. LANDWEBER AND ROBERT E. STONG
}

\begin{abstract}
This paper studies the bilinear form on $H^{j}\left(M ; Z_{2}\right)$ defined by $[x, y]=$ $x \mathrm{Sq}^{2} y[M]$ when $M$ is a closed Spin manifold of dimension $2 j+2$. In analogy with the work of Lusztig, Milnor, and Peterson for oriented manifolds, the rank of this form on integral classes gives rise to a cobordism invariant.
\end{abstract}

1. Introduction. If $M^{2 j+2}$ is a closed Spin manifold of dimension $2 j+2$ one has a symmetric bilinear form

$$
[,]: H^{j}\left(M ; Z_{2}\right) \otimes H^{j}\left(M ; Z_{2}\right) \rightarrow Z_{2}:[x, y]=x \operatorname{Sq}^{2} y[M] .
$$

To see that this form is symmetric, one uses the identity

$$
\begin{aligned}
\left(x \mathrm{Sq}^{2} y+y \mathrm{Sq}^{2} x\right)[M] & =\left(\mathrm{Sq}^{2}(x y)+\mathrm{Sq}^{1} x \mathrm{Sq}^{1} y\right)[M] \\
& =\left(v_{2} x y+v_{1} x \mathrm{Sq}^{1} y\right)[M]=0
\end{aligned}
$$

where $v_{i}$ denotes the $i$ th $\mathrm{Wu}$ class of $M$ and $v_{1}=0=v_{2}$ for Spin manifolds.

The main result of this paper is

Proposition 1.1. For a closed Spin manifold $M^{8 k+2}$ of dimension $8 k+2$ and class $z \in H^{4 k}(M ; Z)$

$$
\rho z \mathrm{Sq}^{2} \rho z[M]=\rho z \mathrm{Sq}^{2} v_{4 k}[M]
$$

where $\rho$ is the $\bmod 2$ reduction and $v_{4 k}$ is the $4 k$ th $W u$ class of $M$.

This result arose in answering a question of Edward Witten, who wished to know the structure of $\Omega_{11}^{\text {Spin }}(K(Z, 4))$. In the process this formula was seen to hold for ten dimensional manifolds.

Considering [, ] as defining a form on integral cohomology via $\rho$, one then has

COROllary 1.2. For a closed Spin manifold $M^{8 k+2}$ of dimension $8 k+2$

$$
w_{4} w_{8 k-2}[M]=v_{4 k} \mathrm{Sq}^{2} v_{4 k}[M]
$$

is the rank modulo 2 of the form [, ] on integral cohomology.

Note. Here, the rank of the form is the dimension as $Z_{2}$ vector space of $H^{4 k}(M ; Z)$ modulo the annihilator of the form.

Received by the editors March 5, 1986 and, in revised form, April 29, 1986.

1980 Mathematics Subject Classification (1985 Pevision). Primary 57R20, 57R90. 
Of course, these results are completely analogous to the work of Lusztig, Milnor, and Peterson [LMP], or originally Browder [B1], on the form $(x, y)=x \operatorname{Sq}^{1} y[M]$ for oriented manifolds of dimension $4 k+1$. The proofs are, unfortunately, rather more complicated, and involve the calculation of the Spin bordism of Eilenberg-Mac Lane spaces just outside the stable range. As a sidelight, this work helps to explain the work of Wilson [W] on the vanishing of Stiefel-Whitney classes in Spin manifolds. Knowledge of the form gives

Corollary 1.3. For a closed Spin manifold $M^{8 k+2}$ of dimension $8 k+2$, the Stiefel-Whitney class $\mathrm{Sq}^{3} v_{4 k}$ is zero.

In $\S 2$, the proof is begun by showing that there is a class $\theta \in H^{*}\left(B \operatorname{Spin} ; Z_{2}\right)$ for which $\rho z \mathrm{Sq}^{2} \rho z[M]=\rho z \cdot \tau^{*}(\theta)[M]$. In $\S 3$, the elementary properties of $\theta$ are described, and in the following section, $\theta$ is shown to be unique by a nasty calculation. $\S 5$ then collects the main results, and the final section contains an extension to mod 4 cohomology suggested by Steven Kahn.

The authors are indebted to Edward Witten, whose questions about the Spin bordism of Eilenberg-Mac Lane spaces led to this work; to Steven Kahn, whose suggestions led to an extension of the results; and to the National Science Foundation for financial support during this work.

2. Spin bordism of Eilenberg-Mac Lane spaces. The basic tool for analyzing the form $[$,$] is given by$

LEMMA 2.1. There are exact sequences

$$
\begin{aligned}
\cdots & \rightarrow \pi_{r+i+1}\left(M \operatorname{Spin} \wedge X_{r}\right) \rightarrow \tilde{\Omega}_{i+j}^{\operatorname{Spin}}(K(Z, j)) \\
& \rightarrow H_{i}(B \operatorname{Spin} ; Z) \rightarrow \pi_{r+i}\left(M \operatorname{Spin} \wedge X_{r}\right) \rightarrow \cdots, \\
\cdots & \rightarrow \pi_{r+i+1}\left(M \operatorname{Spin} \wedge Y_{r}\right) \rightarrow \tilde{\Omega}_{i+j}^{\operatorname{Spin}}\left(K\left(Z_{2}, j\right)\right) \\
& \rightarrow H_{i}\left(B \operatorname{Spin} ; Z_{2}\right) \rightarrow \pi_{r+i}\left(M \operatorname{Spin} \wedge Y_{r}\right) \rightarrow \cdots
\end{aligned}
$$

for $r$ large.

ProOF. One considers the cofibration $\Sigma^{r-j} K(\pi, j) \stackrel{g}{\rightarrow} K(\pi, r) \rightarrow W_{r}$ with $\pi=Z$ or $Z_{2}\left(W_{r}=X_{r}\right.$ or $Y_{r}$, respectively) and $r$ large, and applies reduced Spin bordism. Of course, $\tilde{\Omega}_{r+i}^{\text {Spin }}\left(W_{r}\right)=\pi_{r+i}\left(M\right.$ Spin $\left.\wedge W_{r}\right)$ by interpreting Spin bordism as the homotopy of a spectrum, and $\tilde{\Omega}_{r+i}^{\mathrm{Spin}}\left(\Sigma^{r-j} K(\pi, j)\right)=\tilde{\Omega}_{i+j}^{\mathrm{Spin}}(K(\pi, j))$ using the suspension isomorphism. Finally, for $r$ and $s$ large

$$
\begin{aligned}
\tilde{\Omega}_{r+i}^{\operatorname{Spin}}(K(\pi, r)) & =\pi_{8 s+r+i}\left(M \operatorname{Spin}_{8 s} \wedge K(\pi, r)\right)=\tilde{H}_{8 s+i}\left(M \operatorname{Spin}_{8 s} ; \pi\right) \\
& =H_{i}\left(B \operatorname{Spin}_{8 s} ; \pi\right)=H_{i}(B \operatorname{Spin} ; \pi) .
\end{aligned}
$$

Here $g$ is intended to be the map for which $g^{*} i_{r}=\sigma^{r-j} i_{j}$ with $\sigma$ denoting suspension and $i_{r} \in H^{r}(K(\pi, r) ; \pi)$ being the fundamental class.

In order to analyze the 2-primary part of $\pi_{r+i}\left(M \operatorname{Spin} \wedge W_{r}\right), W_{r}=X_{r}$ or $Y_{r}$, one uses mod 2 cohomology. Heavy use will be made of the structure of $M$ Spin, as described by Anderson, Brown, and Peterson [ABP]. In particular,

$$
\tilde{H}^{*}\left(M \operatorname{Spin} ; Z_{2}\right) \cong\left(\mathscr{A} / \mathscr{A} \mathrm{Sq}^{1}+\mathscr{A} \mathrm{Sq}^{2}\right) U+\left(\mathscr{A} / \mathscr{A} \mathrm{Sq}^{1}+\mathscr{A} \mathrm{Sq}^{2}\right) w_{4}^{2} U
$$


plus terms of higher dimension, where $U$ is the Thom class, $w_{4} \in H^{*}\left(B \operatorname{Spin} ; Z_{2}\right)$ is the universal Stiefel-Whitney class, and $\mathscr{A}$ denotes the mod 2 Steenrod algebra. One then needs to know the structure of $\tilde{H}^{*}\left(W_{r} ; Z_{2}\right)$ as a module over $\mathscr{A}_{1}$, the subalgebra of $\mathscr{A}$ generated by $\mathrm{Sq}^{1}$ and $\mathrm{Sq}^{2}$, which is

$\begin{array}{ccccccc}1 & \mathrm{Sq}^{1} & \mathrm{Sq}^{2} & \mathrm{Sq}^{3}, \mathrm{Sq}^{2} \mathrm{Sq}^{1} & \mathrm{Sq}^{3} \mathrm{Sq}^{1} & \mathrm{Sq}^{5}+\mathrm{Sq}^{4} \mathrm{Sq}^{1} & \mathrm{Sq}^{5} \mathrm{Sq}^{1} \\ \operatorname{dim} 0 & \operatorname{dim} 1 & \operatorname{dim} 2 & \operatorname{dim} 3 & \operatorname{dim} 4 & \operatorname{dim} 5 & \operatorname{dim} 6\end{array}$

From the cofibration

$$
\Sigma^{r-j} K(\pi, j) \stackrel{g}{\rightarrow} K(\pi, r) \stackrel{h}{\rightarrow} W_{r}
$$

one has an exact sequence

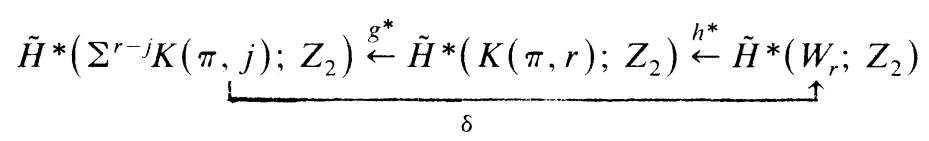

Within the stable range, $\tilde{H}^{*}\left(K(\pi, r) ; Z_{2}\right)$ has a basis given by the classes $\mathrm{Sq}^{K} i_{r}=\mathrm{Sq}^{k_{1}} \ldots \mathrm{Sq}^{k_{s}} i_{r}$ where $K=\left(k_{1}, \ldots, k_{s}\right)$ is an admissible sequence $\left(k_{i} \geqslant\right.$ $\left.2 k_{i+1}\right)$ and, for $\pi=Z, k_{s}>1$. One knows that $H^{*}\left(K(\pi, j) ; Z_{2}\right)$ is the polynomial ring over $Z_{2}$ on the classes $\mathrm{Sq}^{K} i_{j}$ where $K$ is admissible, has $k_{s}>1$ if $\pi=Z$, and has excess $e(K)=\left(k_{1}-2 k_{2}\right)+\left(k_{2}-2 k_{3}\right)+\cdots+\left(k_{s-1}-2 k_{s}\right)+k_{s}$ less than $j$. If $e(K)=j, \mathrm{Sq}^{K} i_{j}=\left(\mathrm{Sq}^{K^{\prime}} i_{j}\right)^{2^{t}}$ for some $K^{\prime}$ and $t$. Since

$$
g^{*}\left(\mathrm{Sq}^{K} i_{r}\right)=\mathrm{Sq}^{K} g^{*}\left(i_{r}\right)=\mathrm{Sq}^{K} \sigma^{r-j} i_{j}=\sigma^{r-j} \mathrm{Sq}^{K} i_{j},
$$

one can readily analyze the kernel and cokernel of $g^{*}$. The kernel of $g^{*}$ has a basis given by the classes $\mathrm{Sq}^{K} i_{r}$ with $e(K)>j$, and the cokernel of $g^{*}$ is given by classes $\sigma^{r-j}\left(\mathrm{Sq}^{k_{1}} i_{j} \cdots \mathrm{Sq}^{k_{t}} i_{j}\right)$ with $t>1$, modulo the classes $\sigma^{r-j}\left(\left(\mathrm{Sq}^{K^{\prime}} i_{j}\right)^{2^{\prime}}\right)$.

As a special case, one can then consider $\pi=Z, j=4 k$, and write down $\tilde{H}^{*}\left(X_{r} ; Z_{2}\right)$ in low dimensions. There is a basis given by

$$
\begin{array}{ll}
\operatorname{dim}(r+4 k+1) & \left\{\mathrm{Sq}^{4 k+1} i_{r}\right\}, \\
\operatorname{dim}(r+4 k+2) & \left\{\mathrm{Sq}^{4 k+2} i_{r}\right\}, \\
\operatorname{dim}(r+4 k+3) & \left\{\mathrm{Sq}^{4 k+3} i_{r}\right\}, \delta \sigma^{r-4 k} i_{4 k} \mathrm{Sq}^{2} i_{4 k}, \\
\operatorname{dim}(r+4 k+4) & \left\{\mathrm{Sq}^{4 k+4} i_{r}\right\},\left\{\mathrm{Sq}^{4 k+3} \mathrm{Sq}^{2} i_{r}\right\}, \delta \sigma^{r-4 k} i_{4 k} \mathrm{Sq}^{3} i_{4 k}
\end{array}
$$

and terms of higher degree. Here $\{x\}$ denotes a class mapping by $h^{*}$ to $x$, i.e. $h^{*}(\{x\})=x$.

Being interested in the action of $\mathscr{A}_{1}$, one needs the Adem relations

$$
\mathrm{Sq}^{1} \mathrm{Sq}^{h}= \begin{cases}\mathrm{Sq}^{b+1}, & b \text { even }>0 \\ 0, & b \text { odd },\end{cases}
$$

and

$$
\mathrm{Sq}^{2} \mathrm{Sq}^{b}=\left\{\begin{array}{ll}
\mathrm{Sq}^{h+2}+\mathrm{Sq}^{h+1} \mathrm{Sq}^{1}, & b \equiv 0,3 \bmod 4, \\
\mathrm{Sq}^{b+1} \mathrm{Sq}^{1}, & b \equiv 1,2 \bmod 4,
\end{array}(b>1)\right.
$$


Then one has $\mathrm{Sq}^{1}\left\{\mathrm{Sq}^{4 k+1} i_{r}\right\}=0, \mathrm{Sq}^{1}\left\{\mathrm{Sq}^{4 k+2} i_{r}\right\}=\left\{\mathrm{Sq}^{4 k+3} i_{r}\right\}$, i.e., $\mathrm{Sq}^{1}\left\{\mathrm{Sq}^{4 k+2} i_{r}\right\}$ is a class which maps to $\mathrm{Sq}^{4 k+3} i_{r}$ and $\left\{\mathrm{Sq}^{4 k+3} i_{r}\right\}$ may be chosen to be $\mathrm{Sq}^{1}$ on the lower class, and

$$
\mathrm{Sq}^{1} \delta \sigma^{r-4 k} i_{4 k} \mathrm{Sq}^{2} i_{4 k}=\delta \sigma^{r-4 k} i_{4 k} \mathrm{Sq}^{3} i_{4 k} .
$$

Also, $\mathrm{Sq}^{2} \mathrm{Sq}^{4 k+1} i_{r}=0$, so there is a $\mu \in Z_{2}$ for which

$$
\mathrm{Sq}^{2}\left\{\mathrm{Sq}^{4 k+1} i_{r}\right\}=\mu \delta \sigma^{r-4 k} i_{4 k} \mathrm{Sq}^{2} i_{4 k} \text {. }
$$

Claim. $\mu \neq 0$. To verify this, one may consider the effect of the assumption that $\mu=0$. To begin, one notices that rationally $\tilde{\Omega}_{8 k}^{\text {Spin }}(K(Z, 4 k))$ has a nonzero class detected by $i_{4 k}^{2}$ which goes to zero in $\tilde{\Omega}_{r+4 k}^{\text {Spin }}(K(Z, r))$, and so $\pi_{r+4 k+1}\left(M\right.$ Spin $\left.\wedge X_{r}\right)$ $=Z+$ torsion. One may then find a map

$$
F \rightarrow M \operatorname{Spin}_{8 s} \wedge X_{r} \stackrel{a}{\rightarrow} K(Z, 8 s+r+4 k+1) \times K\left(Z_{2}, 8 s+r+4 k+2\right)
$$

with $F$ being the fiber, so that

$$
a^{*}\left(i_{8 s+r+4 k+1}\right)=U \cdot\left\{\mathrm{Sq}^{4 k+1} i_{r}\right\}, \quad a^{*}\left(i_{8 s+r+4 k+2}\right)=U \cdot\left\{\mathrm{Sq}^{4 k+2} i_{r}\right\} .
$$

There must then be a class $b \in H^{8 s+r+4 k+2}\left(F, Z_{2}\right)$ transgressing to kill $\mathrm{Sq}^{2} i_{8 s+r+4 k+1}$, with $\mathrm{Sq}^{1} b$ transgressing to $\mathrm{Sq}^{3} i_{8 s+r+4 k+1}$. Thus $\pi_{8 s+r+4 k+2}(F) \cong Z_{2}$ and

$$
\pi_{r+1}\left(M \operatorname{Spin} \wedge X_{r}\right)= \begin{cases}Z, & i=4 k+1 \\ \text { order } 4, & i=4 k+2\end{cases}
$$

modulo odd torsion.

If one now considers the case $k=1$, one has the exact sequence

$$
\begin{array}{ccccc}
\tilde{\Omega}_{10}^{\text {Spin }}(K(Z, 4)) & \stackrel{b}{\rightarrow} & H_{6}(B \text { Spin; } Z) & \rightarrow & \pi_{r+6}\left(M \operatorname{Spin} \wedge X_{r}\right) \\
\| & & Z_{2} & & \\
Z_{2}+Z_{2} & & \tilde{\Omega}_{9}^{\text {Spin }}(K(Z, 4)) & \rightarrow & H_{5}(B \text { Spin; } Z) \\
& \rightarrow & & \| \\
& Z_{2} & & 0
\end{array}
$$

in which the groups $\tilde{\Omega}_{*}^{\text {Spin }}(K(Z, 4))$ are known from [S]. Here $b$ is epic; there is a closed Spin manifold $M^{10}$ and integral class $z \in H^{4}(M ; Z)$ reducing to $w_{4}$ for which $w_{6} \rho z[M]=w_{6} w_{4}[M] \neq 0$. (Note. A specific example of such a manifold is given in [F, p. 218].) Thus $\pi_{r+6}\left(M \operatorname{Spin} \wedge X_{r}\right)=Z_{2}$, and so $\mu=1$ when $k=1$.

One then has a commutative diagram

$$
\begin{array}{ccccc}
H P^{\infty} \wedge \Sigma^{r-4 k} K(Z, 4) & \rightarrow & H P^{\infty} \wedge K(Z, r-4 k+4) & \rightarrow & H P^{\infty} \wedge X_{r} \quad\left(k^{\prime}=1\right) \\
\downarrow \Sigma c & & \downarrow c & & \downarrow d \\
\Sigma^{r-4 k} K(Z, 4 k) & \rightarrow & K(Z, r) & \stackrel{e}{\rightarrow} & X_{r}
\end{array}
$$

in which $c^{*}\left(i_{r}\right)=u^{k-1} i_{r-4 k+4}, u \in H^{4}\left(H P^{\infty} ; Z\right)=Z$ being a generator, with $\Sigma c$ being obtained by suspending the similar map, and with $d$ being the induced map on cofibers.

$$
\begin{aligned}
c^{*} e^{*}\left\{\mathrm{Sq}^{4 k+1} i_{r}\right\} & =\mathrm{Sq}^{4 k+1} c^{*}\left(i_{r}\right) \\
& =u^{2 k-2} \mathrm{Sq}^{5} i_{r-4 k+4}+\text { terms with smaller powers of } u,
\end{aligned}
$$


so

$$
\begin{aligned}
d^{*}\left\{\mathrm{Sq}^{4 k+1} i_{r-4 k+4}\right\}= & u^{2 k-2}\left\{\mathrm{Sq}^{5} i_{r-4 k+4}\right\} \\
& + \text { terms with smaller powers of } u .
\end{aligned}
$$

Since $\mathrm{Sq}^{2} u=0=\mathrm{Sq}^{1} u$, this gives

$$
\begin{aligned}
d^{*}\left(\mathrm{Sq}^{2}\left\{\mathrm{Sq}^{4 k+1} i_{r}\right\}\right)= & u^{2 k-2} \mathrm{Sq}^{2}\left\{\mathrm{Sq}^{5} i_{r-4 k+4}\right\} \\
& + \text { terms with smaller powers of } u .
\end{aligned}
$$

Thus $\mathrm{Sq}^{2}\left\{\mathrm{Sq}^{4 k+1} i_{r}\right\} \neq 0$, and hence $\mu \neq 0$ for all $k$, completing the proof of the claim.

LEMmA 2.2. There is a class $\theta \in H^{4 k+2}\left(B \operatorname{Spin} ; Z_{2}\right)$ for which

$$
\rho z \mathrm{Sq}^{2} \rho z[M]=\tau^{*}(\theta) \rho z[M]
$$

for all Spin $M^{8 k+2}$ and $z \in H^{4 k}(M ; Z)$, where $\tau: M \rightarrow B$ Spin classifies the tangent bundle.

Proof. Consider the diagram

$$
\begin{gathered}
\pi_{r+4 k+3}\left(M \operatorname{Spin} \wedge X_{r}\right) \quad \stackrel{\partial}{\rightarrow} \quad \tilde{\Omega}_{8 k+2}^{\text {Spin }}(K(Z, 4 k)) \rightarrow H_{4 k+2}(B \text { Spin } ; Z) \\
\downarrow \phi \\
Z_{2}
\end{gathered}
$$

where $\phi$ assigns to $f: M^{8 k+2} \rightarrow K(Z, 4 k)$ the characteristic number $f^{*}\left(i_{4 k}\right)$. $\mathrm{Sq}^{2} f^{*}\left(i_{4 k}\right)\left[M^{8 k+2}\right]$. Then $\phi \circ \partial(\alpha)$ is the value on $\alpha$ of the characteristic number $U \cdot \delta \sigma^{r-4 k} i_{4 k} \mathrm{Sq}^{2} i_{4 k}=\mathrm{Sq}^{2}\left(U \cdot\left\{\mathrm{Sq}^{4 k+1} i_{r}\right\}\right)$ and cohomology classes of this form vanish on homotopy $\left(\mathrm{Sq}^{i}\right.$ is zero in a sphere), so $\phi$ is zero on the image of $\partial$.

Now $H_{4 k+2}\left(B\right.$ Spin; $Z$ ) is a $Z_{2}$ vector space and sits inside $H_{4 k+2}\left(B\right.$ Spin; $\left.Z_{2}\right)$, so there is a homomorphism $\psi: H_{4 k+2}\left(B\right.$ Spin; $\left.Z_{2}\right) \rightarrow Z_{2}$ or equivalently class $\theta \in H^{4 k+2}\left(B\right.$ Spin; $\left.Z_{2}\right)$ for which $\psi$ restricts to $\phi$ on the image of $\tilde{\Omega}_{8 k+2}^{\text {Spin }}(K(Z, 4 k))$. Now for $z \in H^{4 k}(M ; Z), \psi\left(\tau_{*}([M] \cap \rho z)\right)=\tau^{*}(\theta) \rho z[M]$ then gives $\phi$ on the class of $(M, z)$, i.e., $\rho z \mathrm{Sq}^{2} \rho z[M]$.

Notice that the proof of the proposition has now been reduced to the identification of the class $\theta$. This will require more work.

3. Describing $\theta$. From the previous section one knows that there is a class $\theta$ in $H^{4 k+2}\left(B \operatorname{Spin} ; Z_{2}\right)$ so that $\tau^{*}(\theta) \rho z[M]=\rho z \operatorname{Sq}^{2} \rho z[M]$ for all $M$ and $z$. One now wishes to find this class.

LEMMA 3.1. The class $\theta$ is only well defined in

$$
H^{4 k+2}\left(B \operatorname{Spin} ; Z_{2}\right) / \mathrm{Sq}^{1} H^{4 k+1}\left(B \operatorname{Spin} ; Z_{2}\right) \text {. }
$$

Proof. For $\eta \in H^{4 k+1}\left(B\right.$ Spin; $\left.Z_{2}\right)$,

$$
\begin{aligned}
\tau^{*}\left(\theta+\mathrm{Sq}^{1} \eta\right) \rho z[M] & =\tau^{*}(\theta) \rho z[M]+\left(\mathrm{Sq}^{1} \tau^{*}(\eta)\right) \cdot \rho z[M] \\
& =\rho z \mathrm{Sq}^{2} \rho z[M]+\left(v_{1} \tau^{*}(\eta) \rho z+\tau^{*}(\eta) \mathrm{Sq}^{1} \rho z\right)[M] \\
& =\rho z \mathrm{Sq}^{2} \rho z[M] .
\end{aligned}
$$

Thus, the class $\theta+\operatorname{Sq}^{1} \eta$ has the same property as does $\theta$. 
Note. This corresponds to the fact that $\tilde{\Omega}_{8 k+2}^{\text {Spin }}(K(Z, 4 k))$ maps into

$$
H_{4 k+2}(B \text { Spin; } Z) \subset H_{4 k+2}\left(B \text { Spin; } Z_{2}\right) \text {, }
$$

with the classes in the image of $\mathrm{Sq}^{1}$ vanishing on integral homology.

LeMma 3.2. $\theta$ is nonzero in $H^{4 k+2}\left(B\right.$ Spin; $\left.Z_{2}\right) / \operatorname{Sq}^{1} H^{4 k+1}\left(B \operatorname{Spin} ; Z_{2}\right)$.

Proof. It is sufficient to exhibit a manifold $M^{8 k+2}$ and integral class $z \in$ $H^{4 k}(M ; Z)$ for which $\rho z \mathrm{Sq}^{2} \rho z[M] \neq 0$. For this one lets $M \subset \mathbf{C} P^{2} \times \mathbf{C} P^{4 k}$ be the Milnor hypersurface dual to $\alpha+\beta, \alpha \in H^{2}\left(\mathbf{C} P^{2} ; Z\right)$ and $\beta \in H^{2}\left(\mathbf{C} P^{4 k} ; Z\right)$ being the generators, and lets $z=\alpha \beta^{2 k-1}$, or more precisely, the pullback to $M$. This is a Spin manifold, and the desired number is nonzero.

Lemma 3.3. $\operatorname{Sq}^{1} \theta \in H^{4 k+3}\left(B \operatorname{Spin} ; Z_{2}\right)$ is a nonzero class with $\tau^{*}\left(\operatorname{Sq}^{1} \theta\right)=0$ in the cohomology of every closed Spin manifold of dimension $8 k+2$. Further, $\theta \in$ $H^{4 k+2}\left(B\right.$ Spin; $\left.Z_{2}\right) / \mathrm{Sq}^{1} H^{4 k+1}\left(B\right.$ Spin; $\left.Z_{2}\right)$ is determined by $\operatorname{Sq}^{1} \theta$.

Proof. According to Anderson, Brown, and Peterson [ABP, Proposition 6.1] $H\left(H^{*}\left(B \operatorname{Spin} ; Z_{2}\right), \mathrm{Sq}^{1}\right)=Z_{2}\left[1 \cdot \mathrm{Sq}^{2^{i}}, P_{j}\right]$ with $i \geqslant 2, j \neq 2^{k}$, is a polynomial ring on generators of dimensions divisible by 4 , so $\mathrm{Sq}^{1}$ maps

$$
H^{4 k+2}\left(B \text { Spin; } Z_{2}\right) / \mathrm{Sq}^{1} H^{4 k+1}\left(B \text { Spin } ; Z_{2}\right)
$$

monomorphically into $H^{4 k+3}\left(B\right.$ Spin; $Z_{2}$ ).

For any closed Spin manifold $M^{8 k+2}$ and class $w \in H^{4 k-1}\left(M ; Z_{2}\right)$ one has

$$
\begin{aligned}
\tau^{*}\left(\mathrm{Sq}^{1} \theta\right) w[M] & =\operatorname{Sq}^{1} \tau^{*}(\theta) w[M] \\
& =\left(v_{1} \tau^{*}(\theta) w+\tau^{*}(\theta) \operatorname{Sq}^{1} w\right)[M]=\tau^{*}(\theta) \rho \beta w[M]
\end{aligned}
$$

where $\beta: H^{4 k-1}\left(M ; Z_{2}\right) \rightarrow H^{4 k}(M ; Z)$ is the Bockstein. Then

$$
\begin{aligned}
\tau^{*}\left(\mathrm{Sq}^{1} \theta\right) w[M] & =\rho \beta w \mathrm{Sq}^{2} \rho \beta w[M]=\mathrm{Sq}^{1} w \cdot \mathrm{Sq}^{2} \mathrm{Sq}^{1} w[M] \\
& =\left(v_{1} w \mathrm{Sq}^{2} \mathrm{Sq}^{1} w+w \cdot \mathrm{Sq}^{1} \mathrm{Sq}^{2} \mathrm{Sq}^{1} w\right)[M]=w \cdot \mathrm{Sq}^{2} \mathrm{Sq}^{2} w[M] \\
& =\left(v_{2} \cdot w \mathrm{Sq}^{2} w+\mathrm{Sq}^{2} w \cdot \mathrm{Sq}^{2} w+\mathrm{Sq}^{1} w \cdot \mathrm{Sq}^{1} \mathrm{Sq}^{2} w\right)[M] \\
& =\left(v_{4 k+1} \mathrm{Sq}^{2} w+v_{1}\left(w \mathrm{Sq}^{1} \mathrm{Sq}^{2} w\right)\right)[M]
\end{aligned}
$$

and $v_{1}=0=v_{4 k+1}$ in $M$, so this is zero. By Poincaré duality, this gives $\tau^{*}\left(\operatorname{Sq}^{1} \theta\right)=$ 0 .

Note. Because $H^{7}\left(B\right.$ Spin; $\left.Z_{2}\right)=Z_{2}$, for $k=1$ one has $\operatorname{Sq}^{1} \theta=w_{7}$, and has Wilson's result $[\mathbf{W}]$ that $w_{7}$ is zero in every 10 dimensional Spin manifold. Also $w_{7}=\mathrm{Sq}^{3} v_{4}$ and $\theta=\mathrm{Sq}^{2} v_{4} \in H^{6}\left(B \operatorname{Spin} ; Z_{2}\right)=Z_{2}$.

4. A calculation. One now turns attention to the cofibration (for $k \geqslant 2$ )

$$
\Sigma^{r-4 k} K\left(Z_{2}, 4 k\right) \stackrel{g}{\rightarrow} K\left(Z_{2}, r\right) \stackrel{h}{\rightarrow} Y_{r}
$$

with $r$ large, and may write down $\tilde{H}^{*}\left(Y_{r} ; Z_{2}\right)$. The kernel of $g^{*}$ has a basis given by the classes $\mathrm{Sq}^{I} i_{r}$ with $I$ admissible and having excess greater than $4 k$, and writing $\sigma$ for $\sigma^{r-4 k}, i$ for $i_{4 k}$, the kernel of $h^{*}$ or image of $\delta$ has a basis given by classes $\delta \sigma \mathrm{Sq}^{I_{1}} i \cdots \mathrm{Sq}^{I_{s}} i$ for which the $I_{j}$ are admissible, have excess less than $4 k$, and for which $s>1$ and $\left(I_{1}, \ldots, I_{s}\right) \neq(J, \ldots, J)$ with $2^{t} J$ 's, $t>0$; i.e., not the $2^{t}$ th power of an indecomposable. 
In order to study $\tilde{H}^{*}\left(M \operatorname{Spin}_{8 s} \wedge Y_{r} ; Z_{2}\right)$, one recalls that $\tilde{H}^{*}\left(M \operatorname{Spin}_{8 s} ; Z_{2}\right)$ is a free $\mathscr{A} / \mathscr{A} \mathrm{Sq}^{1}+\mathscr{A} \mathrm{Sq}^{2}$ module on $U$ and $w_{4}^{2} U$ in dimensions $8 s$ and $8 s+8$ with additional generators in dimension $8 s+10$ and higher. Here $s$ is to be large.

Because $\tilde{\Omega}_{*}^{\text {Spin }}\left(K\left(Z_{2}, 4 k\right)\right)$ and $H_{*}\left(B\right.$ Spin; $\left.Z_{2}\right)$ are purely 2-primary, so is $\pi_{*}\left(M \operatorname{Spin}_{8 s} \wedge Y_{r}\right)$. If one then examines the Bockstein spectral sequence for $\tilde{H}^{*}\left(M \operatorname{Spin}_{8 s} \wedge Y_{r} ; Z_{2}\right)($ see $[\mathrm{B} 2])$, then

$$
E_{1}=\tilde{H}^{*}\left(M \operatorname{Spin}_{8 s} \wedge Y_{r} ; Z_{2}\right), \quad d_{1}=\mathrm{Sq}^{1}
$$

and $E^{\infty}$ is zero since $\tilde{H}^{*}\left(M \operatorname{Spin}_{8 s} \wedge Y_{r} ; Z\right)$ consists entirely of torsion. Thus all classes in $\mathrm{ker} \mathrm{Sq}^{1} / \mathrm{im} \mathrm{Sq}^{1}$ are related by higher order Bocksteins.

One may begin by finding a map

$$
M \operatorname{Spin}_{8 s} \wedge Y_{r} \stackrel{f_{1}}{\rightarrow} K\left(Z_{2^{\prime}}, 8 s+r+4 k+1\right)
$$

for which

$$
f_{1}^{*}\left(i_{8 s+r+4 k+1}\right)=U\left\{\mathrm{Sq}^{4 k+1} i_{r}\right\},
$$

where $\{x\}$ denotes some class with $h^{*}\{x\}=x$, and for which $f_{1}^{*}\left(\beta i_{8 s+r+4 k+1}\right), \beta$ being the Bockstein operation, is a nonzero class in the kernel of $\mathrm{Sq}^{1}$. Of course, if $t=1, \beta=\mathrm{Sq}^{1}$. Since $\mathrm{Sq}^{1} \mathrm{Sq}^{4 k+2} i_{r}=\mathrm{Sq}^{4 k+3} i_{r} \neq 0$, one must have $f_{1}^{*}\left(\beta i_{8 s+r+4 k+1}\right)$ $=U \delta \sigma i \mathrm{Sq}^{1} i$.

LEMMA 4.1. $t=1$.

Proof. Clearly

$$
Z_{2^{i}}=\pi_{8 s+r+4 k+1}\left(M \operatorname{Spin}_{8 s} \wedge Y_{r}\right) \cong \pi_{8 s+r+4 k+1}\left(S^{8 s} \wedge Y_{r}\right)
$$

is the bottom stable homotopy group. Applying stable homotopy to the cofibration gives an exact sequence

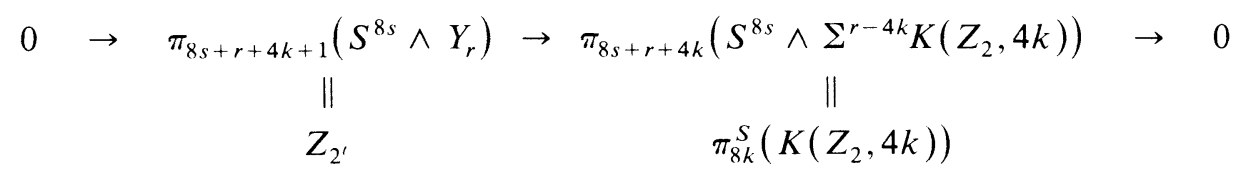

and according to Brown [B3, Lemma (1.2)], the stable homotopy group of $K\left(Z_{2}, 4 k\right)$ is $Z_{2}$.

Because $M \operatorname{Spin}_{8 s}$ is a product (corresponding to the decomposition of cohomology) there is also a map

$$
\operatorname{MSpin}_{8 s} \wedge Y_{r} \underset{\tilde{f}_{1}}{\rightarrow} K\left(Z_{2}, 8 s+r+4 k+9\right)
$$

for which

$$
\tilde{f}_{1}^{*}\left(i_{8 s+r+4 k+9}\right)=w_{4}^{2} U\left\{\mathrm{Sq}^{4 k+1} i_{r}\right\}
$$

and

$$
f_{1}^{*}\left(\mathrm{Sq}^{1} i_{8 s+r+4 k+9}\right)=w_{4}^{2} U \delta \sigma i \mathrm{Sq}^{1} i
$$


Note. This is the only class in the range up to dimension $8 s+r+4 k+9$ involving the generator $w_{4}^{2} U$.

One then has $h^{*} f_{1}^{*}$ sending $\mathrm{Sq}^{2} i_{8 s+r+4 k+1}$ to $U \mathrm{Sq}^{4 k+2} \mathrm{Sq}^{1} i_{r}, \mathrm{Sq}^{3} i_{8 s+r+4 k+1}$ to $U \mathrm{Sq}^{4 k+3} \mathrm{Sq}^{1} i_{r}$ and $\mathrm{Sq}^{2} \mathrm{Sq}^{3} i_{8 s+r+4 k+1}$ to $U \mathrm{Sq}^{4 k+5} \mathrm{Sq}^{1} i_{r}$. Also, under $f_{1}^{*} \mathrm{Sq}^{2} \mathrm{Sq}^{1} i_{8 s+r+4 k+1}$ goes to $U \delta \sigma i \mathrm{Sq}^{2} \mathrm{Sq}^{1} i+U \delta \sigma \mathrm{Sq}^{1} i \mathrm{Sq}^{2} i, \mathrm{Sq}^{3} \mathrm{Sq}^{1} i_{8 s+r+4 k+1}$ goes to

$$
U \delta \sigma \mathrm{Sq}^{1} i \mathrm{Sq}^{2} i+U \delta \sigma \mathrm{Sq}^{1} i \mathrm{Sq}^{2} \mathrm{Sq}^{1} i+U \delta \sigma i \mathrm{Sq}^{3} i \mathrm{Sq}^{1} i,
$$

and $\mathrm{Sq}^{2} \mathrm{Sq}^{3} \mathrm{Sq}^{1} i_{8 s+r+4 k+1}$ goes to

$$
\begin{array}{r}
U \delta \sigma i \mathrm{Sq}^{5} \mathrm{Sq}^{1} i+U \delta \sigma \mathrm{Sq}^{2} i \mathrm{Sq}^{3} \mathrm{Sq}^{1} i+U \delta \sigma \mathrm{Sq}^{3} i \mathrm{Sq}^{2} \mathrm{Sq}^{1} i \\
+U \delta \sigma \mathrm{Sq}^{1} i \mathrm{Sq}^{5} i+U \delta \sigma \mathrm{Sq}^{1} i \mathrm{Sq}^{4} \mathrm{Sq}^{1} i .
\end{array}
$$

Because the action of $\mathscr{A}$ on $U$ gives a free $\mathscr{A} / \mathscr{A} \mathrm{Sq}^{1}+\mathscr{A} \mathrm{Sq}^{2}$ module, one then sees that $f_{1}^{*}$ is monic.

One may now find maps $f_{2}: M \operatorname{Spin}_{8 s} \wedge Y_{r} \rightarrow K\left(Z_{2}, 8 s+r+4 k+2\right)$ and $f_{3}$ : $M_{\operatorname{Spin}_{8 s}} \wedge Y_{r} \rightarrow K\left(Z_{2}, 8 s+r+4 k+3\right)$ for which $f_{2}^{*}\left(i_{8 s+r+4 k+2}\right)=$ $U\left\{\mathrm{Sq}^{4 k+2} i_{r}\right\}$, where $\left\{\mathrm{Sq}^{4 k+2} i_{r}\right\}$ is some class mapping to $\mathrm{Sq}^{4 k+2} i_{r}$ under $h^{*}$ and $f_{3}^{*}\left(i_{8 s+r+4 k+3}\right)=U \delta \sigma i \mathrm{Sq}^{2} i$.

Now

$$
h^{*} f_{2}^{*}\left(\mathrm{Sq}^{1} i_{8 s+r+4 k+2}\right)=U \mathrm{Sq}^{4 k+3} i_{r}
$$

$\cdots+1$

$$
h^{*} f_{2}^{*}\left(\mathrm{Sq}^{2} i_{8 s+r+4 k+2}\right)=U \mathrm{Sq}^{4 k+3} \mathrm{Sq}^{1} i_{r}=h^{*} f_{1} *\left(\mathrm{Sq}^{3} i_{8 s+r+4 k+1}\right) .
$$

Thus

$$
\begin{aligned}
f_{1}^{*}\left(\mathrm{Sq}^{3} i_{8 s+r+4 k+1}\right)+ & f_{2}^{*}\left(\mathrm{Sq}^{2} i_{8 s+r+4 k+2}\right) \\
& =\lambda U \delta \sigma i \mathrm{Sq}^{3} i+\mu U \delta \sigma i \mathrm{Sq}^{2} \mathrm{Sq}^{1} i+\nu U \delta \sigma \mathrm{Sq}^{1} i \mathrm{Sq}^{2} i
\end{aligned}
$$

for some $\lambda, \mu, \nu \in Z_{2}$. One now applies $\mathrm{Sq}^{3}$ to this relation, using the fact that $\mathrm{Sq}^{3} \mathrm{Sq}^{2}=0$ to obtain

$$
\begin{aligned}
U \delta \sigma i \mathrm{Sq}^{5} \mathrm{Sq}^{1} i+U \delta & \mathrm{Sq}^{2} i \mathrm{Sq}^{3} \mathrm{Sq}^{1} i+U \delta \sigma \mathrm{Sq}^{3} i \mathrm{Sq}^{2} \mathrm{Sq}^{1} i \\
& +U \delta \sigma \mathrm{Sq}^{1} i \mathrm{Sq}^{5} i+U \delta \sigma \mathrm{Sq}^{1} i \mathrm{Sq}^{4} \mathrm{Sq}^{1} i \\
= & \lambda\left(U \delta \sigma \mathrm{Sq}^{1} i\left(\mathrm{Sq}^{5}+\mathrm{Sq}^{4} \mathrm{Sq}^{1}\right) i+U \delta \sigma i \mathrm{Sq}^{5} \mathrm{Sq}^{1} i\right) \\
& +\mu\left(U \delta \sigma \mathrm{Sq}^{3} i \mathrm{Sq}^{2} \mathrm{Sq}^{1} i+U \delta \sigma \mathrm{Sq}^{2} i \mathrm{Sq}^{3} \mathrm{Sq}^{1} i\right) \\
& +\nu\left(U \delta \sigma \mathrm{Sq}^{2} i \mathrm{Sq}^{3} \mathrm{Sq}^{1} i+U \delta \sigma \mathrm{Sq}^{2} \mathrm{Sq}^{1} i \mathrm{Sq}^{3} i\right)
\end{aligned}
$$

so $\lambda=1=\mu+\nu$. One also has

$$
\begin{aligned}
a f_{1}^{*} & \left(\mathrm{Sq}^{2} \mathrm{Sq}^{1} i_{8 s+r+4 k+1}\right)+b f_{3} *\left(\mathrm{Sq}^{1} i_{8 s+r+4 k+3}\right) \\
\quad= & b U \delta \sigma i \mathrm{Sq}^{3} i+a U \delta \sigma i \mathrm{Sq}^{2} \mathrm{Sq}^{1} i+(a+b) U \delta \sigma \mathrm{Sq}^{1} i \mathrm{Sq}^{2} i
\end{aligned}
$$

so that proper choice of $a$ and $b$ gives all possible $\lambda, \mu, \nu$ with $\lambda+\mu+\nu=0$. Thus, one has a relation

(*) $\quad f_{1}^{*}\left(\mathrm{Sq}^{3} i_{8 s+r+4 k+1}+\mu \mathrm{Sq}^{2} \mathrm{Sq}^{1} i_{8 s+r+4 k+1}\right)+f_{2}^{*}\left(\mathrm{Sq}^{2} i_{8 s+r+4 k+2}\right)$

$$
+f_{3}^{*}\left(\mathrm{Sq}^{1} i_{8 s+r+4 k+3}\right)=0 \text {. }
$$


For convenience, one lets

$$
\xi=\mathrm{Sq}^{3} i_{8 s+r+4 k+1}+\mu \mathrm{Sq}^{2} \mathrm{Sq}^{1} i_{8 s+r+4 k+1}+\mathrm{Sq}^{2} i_{8 s+r+4 k+2}+\mathrm{Sq}^{1} i_{8 s+r+4 k+3}
$$

in the cohomology of the product of Eilenberg-Mac Lane spaces. One now continues to describe the homomorphism. Applying $h^{*} f_{2}^{*}$ to $\mathrm{Sq}^{2} \mathrm{Sq}^{1} i_{8 s+r+4 k+2}$ gives $U$. $\mathrm{Sq}^{4 k+5} i_{r}+U \cdot \mathrm{Sq}^{4 k+4} \mathrm{Sq}^{1} i_{r}$, and all other operations $\gamma i_{8 s+r+4 k+2}$ with $\gamma \in \mathscr{A}_{1}$ actually lie in $\mathscr{A}_{1} \mathrm{Sq}^{2}$, so that

$$
\begin{aligned}
\xi & =\mathrm{Sq}^{2} i_{8 s+r+4 k+2}+\cdots, \\
\mathrm{Sq}^{1} \xi & =\mathrm{Sq}^{3} i_{8 s+r+4 k+2}+\cdots, \\
\mathrm{Sq}^{2} \xi & =\mathrm{Sq}^{3} \mathrm{Sq}^{1} i_{8 s+r+4 k+2}+\cdots, \\
\mathrm{Sq}^{2} \mathrm{Sq}^{1} \xi & =\left(\mathrm{Sq}^{5}+\mathrm{Sq}^{4} \mathrm{Sq}^{1}\right) i_{8 s+r+4 k+2}+\cdots, \\
\mathrm{Sq}^{3} \mathrm{Sq}^{1} \xi & =\mathrm{Sq}^{5} \mathrm{Sq}^{1} i_{8 s+r+4 k+2}+\cdots .
\end{aligned}
$$

Applying $f_{3}^{*}$ to $\mathrm{Sq}^{1} i_{8 s+r+4 k+3}$ gives $U \delta \sigma i \mathrm{Sq}^{3} i+U \delta \sigma \mathrm{Sq}^{1} i \mathrm{Sq}^{2} i$, a fact used above without mention, $\mathrm{Sq}^{2} i_{8 s+r+4 k+3}$ gives $U \delta \sigma i \mathrm{Sq}^{3} \mathrm{Sq}^{1} i+U \delta \sigma \mathrm{Sq}^{1} i \mathrm{Sq}^{3} i, \mathrm{Sq}^{3} i_{8 s+r+4 k+3}$ gives $U \delta \sigma \mathrm{Sq}^{1} i \mathrm{Sq}^{3} \mathrm{Sq}^{1} i, \mathrm{Sq}^{2} \mathrm{Sq}^{1} i_{8 s+r+4 k+3}$ gives

$$
\begin{gathered}
U \delta \sigma \mathrm{Sq}^{2} i \mathrm{Sq}^{3} i+U \delta \sigma i \mathrm{Sq}^{5} i+U \delta \sigma i \mathrm{Sq}^{4} \mathrm{Sq}^{1} i \\
+U \delta \sigma \mathrm{Sq}^{2} i \mathrm{Sq}^{2} \mathrm{Sq}^{1} i+U \delta \sigma \mathrm{Sq}^{1} i \mathrm{Sq}^{3} \mathrm{Sq}^{1} i,
\end{gathered}
$$

and $\mathrm{Sq}^{2} \mathrm{Sq}^{3} i_{8 s+r+4 k+3}=\left(\mathrm{Sq}^{5}+\mathrm{Sq}^{4} \mathrm{Sq}^{1}\right) i_{8 s+r+4 k+3}$ gives $U \delta \sigma \mathrm{Sq}^{2} \mathrm{Sq}^{1} i \mathrm{Sq}^{3} \mathrm{Sq}^{1} i+$ $U \delta \sigma \mathrm{Sq}^{1} i \mathrm{Sq}^{5} \mathrm{Sq}^{1} i$. Finally, $\mathrm{Sq}^{3} \mathrm{Sq}^{1} i_{8 s+r+4 k+3}$ goes to

$$
\begin{array}{rl}
U \delta \sigma \mathrm{Sq}^{2} & i \mathrm{Sq}^{3} \mathrm{Sq}^{1} i+U \delta \sigma \mathrm{Sq}^{2} \mathrm{Sq}^{1} i \mathrm{Sq}^{3} i+U \delta \sigma \mathrm{Sq}^{1} i \mathrm{Sq}^{5} i \\
& +U \delta \sigma \mathrm{Sq}^{1} i \mathrm{Sq}^{4} \mathrm{Sq}^{1} i+U \delta \sigma i \mathrm{Sq}^{5} \mathrm{Sq}^{1} i \\
= & f_{1}^{*}\left(\mathrm{Sq}^{2} \mathrm{Sq}^{3} \beta i_{8 s+r+4 k+1}\right)
\end{array}
$$

and $\mathrm{Sq}^{5} \mathrm{Sq}^{1} i_{8 s+r+4 k+3}$ goes to zero.

One then notices that

$$
\mathrm{Sq}^{3} \xi=\mathrm{Sq}^{5} \mathrm{Sq}^{1} i_{8 s+r+4 k+1}+\mathrm{Sq}^{3} \mathrm{Sq}^{1} i_{8 s+r+4 k+3}
$$

and

$$
\mathrm{Sq}^{2} \mathrm{Sq}^{3} \xi=\mathrm{Sq}^{5} \mathrm{Sq}^{1} i_{8 s+r+4 k+3}
$$

giving the two relations which just occurred. One then observes that the map

$$
\begin{gathered}
\operatorname{MSpin}_{8 s} \wedge Y_{r} \stackrel{f_{1} \times f_{2} \times f_{3}}{\rightarrow} K\left(Z_{2}, 8 s+r+4 k+1\right) \times K\left(Z_{2}, 8 s+r+4 k+2\right) \\
\times K\left(Z_{2}, 8 s+r+4 k+3\right)
\end{gathered}
$$

has kernel in mod 2 cohomology generated over $\mathscr{A}$ by $\xi$.

One now has a map

$$
f_{4} \times f_{4}^{\prime}: M \operatorname{Spin}_{8 s} \wedge Y_{r} \rightarrow K\left(Z_{2}, 8 s+r+4 k+4\right) \times K\left(Z_{2}, 8 s+r+4 k+4\right)
$$
with $f_{4}^{*}\left(i_{8 s+r+4 k+4}\right)=U\left\{\mathrm{Sq}^{4 k+4} i_{r}\right\}$ and $f_{4}^{\prime *}\left(i_{8 s+r+4 k+4}^{\prime}\right)=U \delta \sigma \mathrm{Sq}^{1} i \mathrm{Sq}^{2} i$ so that

$$
h^{*} f_{4}^{*}\left(\mathrm{Sq}^{1} i_{8 s+r+4 k+4}\right)=U \mathrm{Sq}^{4 k+5} i_{r}
$$


and

$$
f_{4}^{\prime *}\left(\mathrm{Sq}^{1} i_{8 s+r+4 k+4}^{\prime}\right)=U \delta \sigma \mathrm{Sq}^{1} i \mathrm{Sq}^{3} i \text {. }
$$

This brings one to dimension $8 s+r+4 k+5$ in which questionable behavior occurs. No class described so far hits $U \cdot \mathrm{Sq}^{4 k+3} \mathrm{Sq}^{2} i_{r}$ in $M \operatorname{Spin}_{8 s} \wedge K\left(Z_{2}, r\right)$ and $\mathrm{Sq}^{1}\left(U \cdot \mathrm{Sq}^{4 k+3} \mathrm{Sq}^{2} i_{r}\right)=0$. One may choose a class $\left\{\mathrm{Sq}^{4 k+3} \mathrm{Sq}^{2} i_{r}\right\}=x$ and $\mathrm{Sq}^{1} x$ will lie in the image of $\delta$, and also in the kernel of $\mathrm{Sq}^{1}$. Thus $\mathrm{Sq}^{1} x$ is a linear combination of

$$
\begin{gathered}
\delta \sigma i \mathrm{Sq}^{5} i+\delta \sigma \mathrm{Sq}^{1} i \mathrm{Sq}^{4} i=\mathrm{Sq}^{1}\left(\delta \sigma i \mathrm{Sq}^{4} i\right), \\
\delta \sigma \mathrm{Sq}^{1} i \mathrm{Sq}^{3} \mathrm{Sq}^{1} i=\mathrm{Sq}^{1}\left(\delta \sigma i \mathrm{Sq}^{3} \mathrm{Sq}^{1} i\right),
\end{gathered}
$$

and $\delta \sigma \mathrm{Sq}^{2} i \mathrm{Sq}^{3} i$. By changing $x$ to some $x+a \delta \sigma i \mathrm{Sq}^{4} i+b \delta \sigma i \mathrm{Sq}^{3} \mathrm{Sq}^{1} i$, one may assume that $\mathrm{Sq}^{1} x=c \delta \sigma \mathrm{Sq}^{2} i \mathrm{Sq}^{3} i$. If $c \neq 0$, one may let $f_{5}: M \operatorname{Spin}_{8 s} \wedge Y \rightarrow$ $K\left(Z_{2}, 8 s+r+4 k+5\right)$ with $f_{5}^{*}\left(i_{8 s+r+4 k+5}\right)=U \cdot x$ and then $f_{5}{ }^{*}\left(\mathrm{Sq}^{1} i_{8 s+r+4 k+5}\right)$ $=U \delta \sigma \mathrm{Sq}^{2} i \mathrm{Sq}^{3} i$. If $c=0$, then $x$ represents a nonzero class in $\mathrm{ker} \mathrm{Sq}^{1} / \mathrm{im} \mathrm{Sq}^{1}$. There is then a higher-order Bockstein $\beta$ defined on $x$ so that $\beta x$ represents a nonzero class in $\left(\mathrm{ker} \mathrm{Sq}^{1} / \mathrm{im} \mathrm{Sq}^{1}\right)_{r+4 k+6}$. Because $\mathrm{Sq}^{4 k+5} \mathrm{Sq}^{1} i_{r}=\mathrm{Sq}^{1} \mathrm{Sq}^{4 k+4} \mathrm{Sq}^{1} i_{r}$, $\mathrm{Sq}^{1} \mathrm{Sq}^{4 k+6} i_{r}=\mathrm{Sq}^{4 k+7} i_{r}$ and $\mathrm{Sq}^{1} \mathrm{Sq}^{4 k+4} \mathrm{Sq}^{2} i_{r}=\mathrm{Sq}^{4 k+5} \mathrm{Sq}^{2} i_{r}$, and the facts on $\mathrm{Sq}^{1}$ for the image of $\delta$, this group is $Z_{2}$ with generator $\delta \sigma \mathrm{Sq}^{1} i \mathrm{Sq}^{3} i$. Since $U$ is an integral class, one can find a map $f_{5}: M \operatorname{Spin}_{8 s} \wedge Y_{r} \rightarrow K\left(Z_{2} v, 8 s+r+4 k+5\right)$ for which $f_{5}^{*}\left(i_{8 s+r+4 k+5}\right)=U \cdot x$ for which $f_{5}^{*}\left(\beta i_{8 s+r+4 k+5}\right)=U \delta \sigma \mathrm{Sq}^{2} i \mathrm{Sq}^{3} i$ modulo the image of $\mathrm{Sq}^{1}$. By allowing the possibility that $v=1$, one may use this description to cover the $c \neq 0$ case as well, giving a map

$$
f_{5}: \operatorname{MSpin}_{8 s} \wedge Y_{r} \rightarrow K\left(Z_{2} v, 8 s+r+4 k+5\right)
$$

with $f_{5}^{*}\left(i_{8 s+r+4 k+5}\right)=U \cdot\left\{\mathrm{Sq}^{4 k+4} i_{r}\right\}$ and $f_{5}^{*}\left(\beta i_{8 s+r+4 k+5}\right)=U \delta \sigma \mathrm{Sq}^{2} i \mathrm{Sq}^{3} i$ modulo an appropriate term.

One also has a map $f_{5}^{\prime}: \operatorname{MSpin}_{8 s} \wedge Y_{r} \rightarrow K\left(Z_{2}, 8 s+r+4 k+5\right)$ for which $f_{5}^{\prime *}\left(i_{8 s+r+4 k+5}^{\prime}\right)=U \delta \sigma i \mathrm{Sq}^{4} i$. Similarly, in higher dimensions one can find maps into Eilenberg-Mac Lane spaces $K\left(Z_{2}, 8 s+r+4 k+i\right)$ for which

$$
\begin{aligned}
& i=6: \quad f_{6}^{*}\left(i_{8 s+r+4 k+6}\right)=U\left\{\mathrm{Sq}^{4 k+4} \mathrm{Sq}^{2} i_{r}\right\} \text {, } \\
& f_{6}^{\prime *}\left(i_{8 s+r+4 k+6}^{\prime}\right)=U \delta \sigma i \mathrm{Sq}^{5} i \text {, } \\
& i=7: \quad f_{7}^{*}\left(i_{8 s+r+4 k+7}\right)=U\left\{\mathrm{Sq}^{4 k+4} \mathrm{Sq}^{2} \mathrm{Sq}^{1} i_{r}\right\} \text {, } \\
& f_{7}^{\prime *}\left(i_{8 s+r+4 k+7}^{\prime}\right)=U \delta \sigma i \mathrm{Sq}^{6} i \text {, } \\
& f_{7}^{\prime \prime *}\left(i_{8 s+r+4 k+7}^{\prime \prime}\right)=U \delta \sigma i \mathrm{Sq}^{5} \mathrm{Sq}^{1} i \text {, } \\
& f_{7}^{\prime \prime \prime} *\left(i_{8 s+r+4 k+7}^{\prime \prime \prime}\right)=U \delta \sigma i \mathrm{Sq}^{4} \mathrm{Sq}^{2} i \text {, } \\
& i=8: \quad f_{8}^{*}\left(i_{8 s+r+4 k+8}\right)=U\left\{\mathrm{Sq}^{4 k+8} i_{r}\right\} \text {, }
\end{aligned}
$$

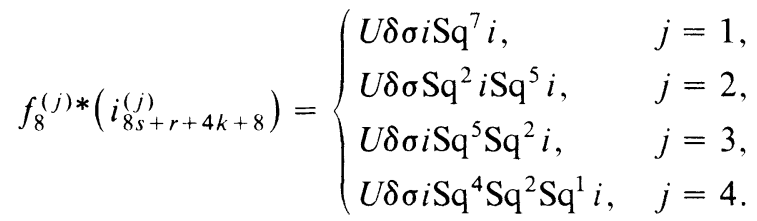


By tedious and unpleasant calculation, one may then verify that the product of all of these maps

$$
f: \operatorname{Spin}_{8 s} \wedge Y_{r} \rightarrow \prod_{i=1}^{8} K\left(G_{i}, 8 s+r+4 k+i\right)
$$

where

\begin{tabular}{c|c|c|c|c|c|c|c|c}
$i$ & 1 & 2 & 3 & 4 & 5 & 6 & 7 & 8 \\
\hline$G_{i}$ & $Z_{2}$ & $Z_{2}$ & $Z_{2}$ & $2 Z_{2}$ & $Z_{2} v+Z_{2}$ & $2 Z_{2}$ & $4 Z_{2}$ & $5 Z_{2}$
\end{tabular}

induces an epimorphism in mod 2 cohomology through dimension $8 s+r+4 k+8$, and that through dimension $8 s+r+4 k+9$ the kernel is generated over $\mathscr{A}$ by $\xi$. One may then choose a minimal set of additional generators in dimension $8 s+r+$ $4 k+9$, giving

$$
\hat{f}: \operatorname{Spin}_{8 s} \wedge Y_{r} \rightarrow \prod_{i=1}^{9} K\left(G_{i}, 8 s+r+4 k+i\right)
$$

so that $\hat{f}^{*}$ is epic through dimension $8 s+r+4 k+9$, and has kernel generated by $\xi$ over $\mathscr{A}$ through this dimension.

Letting $F$ be the fiber of $\hat{f}$, one then has a fibration

$$
F \rightarrow M \operatorname{Spin}_{8 s} \wedge Y_{r} \stackrel{\hat{f}}{\rightarrow} \prod_{i=1}^{9} K\left(G_{i}, 8 s+r+4 k+i\right)
$$

and may calculate

$$
\begin{aligned}
\tilde{H}^{*}\left(F ; Z_{2}\right) \cong & \mathscr{A} / \mathscr{A} \mathrm{Sq}^{5} \mathrm{Sq}^{1} j_{8 s+r+4 k+3} \\
& + \text { terms of dimension } 8 s+r+4 k+9 \text { or higher }
\end{aligned}
$$

where $j_{8 s+r+4 k+3}$ transgresses to $\xi$. The map $e: F \rightarrow K\left(Z_{2}, 8 s+r+4 k+3\right)$ with $e^{*}\left(i_{8 s+r+4 k+3}\right)=j_{8 s+r+4 k+3}$ induces an isomorphism in mod 2 cohomology in dimension less than or equal to $8 s+r+4 k+8$. Thus $e$ induces an isomorphism in homotopy through dimension $8 s+r+4 k+7$ and is epic in dimension $8 s+r+$ $4 k+8$ (which is obvious).

One may now read off the homotopy groups to obtain

LEMMA 4.2. For $j=4 k$ with $k \geqslant 1$,

$$
\pi_{r+4 k+7}\left(M \operatorname{Spin} \wedge Y_{r}\right)=Z_{2}+Z_{2}+Z_{2}+Z_{2}
$$

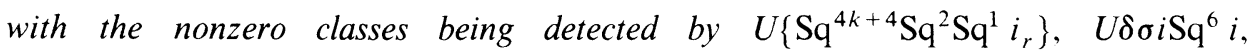
$U \delta \sigma i \mathrm{Sq}^{5} \mathrm{Sq}^{1} i$, and $U \delta \sigma i \mathrm{Sq}^{4} \mathrm{Sq}^{2} i$. In addition, there is a class in $\pi_{r+4 k+3}\left(M \operatorname{Spin} \wedge Y_{r}\right)$ which is detected by $U \delta \sigma i \mathrm{Sq}^{2} i$.

Note. The class in $\pi_{r+4 k+3}\left(M\right.$ Spin $\left.\wedge Y_{r}\right)$ also occurs for $k=1$, since for $k=1$, the description of $\tilde{H}^{*}\left(Y_{r}, Z_{2}\right)$ is correct through dimension $r+4 k+5$, the first problem being the class $\delta \sigma i \mathrm{Sq}^{5} i$. 
Proof. One has

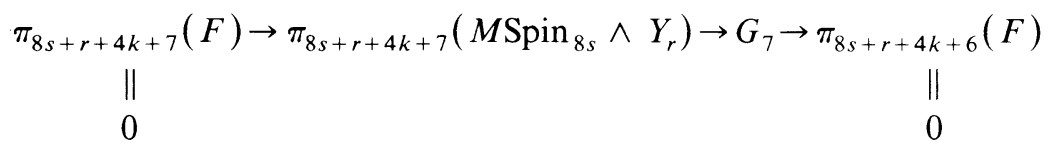

and

$$
\pi_{8 s+r+4 k+3}\left(M \operatorname{Spin}_{8 s} \wedge Y_{r}\right) \rightarrow G_{3} \quad \rightarrow \quad \pi_{8 s+r+4 k+2}(F)
$$

5. The main results. Having done all the hard work, one can now obtain

Proposition 5.1. For a closed Spin manifold $M^{8 k+2}$ of dimension $8 k+2$ and class $z \in H^{4 k}(M ; Z), \rho z \mathrm{Sq}^{2} \rho z[M]=\rho z \mathrm{Sq}^{2} v_{4 k}[M]$.

Proof. For $k=1, \theta=\mathrm{Sq}^{2} v_{4}$ is the only nonzero class in $H^{6}\left(B \operatorname{Spin} ; Z_{2}\right)$. Assuming $k \geqslant 3, \mathrm{Sq}^{1} \theta \in H^{4 k+3}\left(B \operatorname{Spin} ; Z_{2}\right)$ is zero in every Spin manifold of dimension $8 k+2$ and hence in every Spin manifold of smaller dimension. If one considers the sequence

$$
\begin{aligned}
\tilde{\Omega}_{8 k-1}^{\text {Spin }}\left(K\left(Z_{2}, 4 k-4\right)\right) & \stackrel{g}{\rightarrow} H_{4 k+3}\left(B \operatorname{Spin} ; Z_{2}\right) \stackrel{h}{\rightarrow} \pi_{r+4(k-1)+7}\left(M \operatorname{Spin} \wedge Y_{r}\right) \\
& \stackrel{\partial}{\rightarrow} \tilde{\Omega}_{8 k-2}^{\text {Spin }}\left(K\left(Z_{2}, 4 k-4\right)\right),
\end{aligned}
$$

then $k-1 \geqslant 2$ and $\pi_{r+4(k-1)+7}\left(M \operatorname{Spin} \wedge Y_{r}\right)=4 Z_{2}$. The classes detected by $U \delta \sigma i \mathrm{Sq}^{6} i, U \delta \sigma i \mathrm{Sq}^{5} i$, and $U \delta \sigma i \mathrm{Sq}^{4} \mathrm{Sq}^{2} i$ map nontrivially under $\partial$, i.e. the value of $U \delta \sigma y$ on $a$ is the value of $y$ on $\partial a$. Thus, the image of $h$ or cokernel of $g$ is at most $Z_{2}$ and is detected by $U\left\{\mathrm{Sq}^{4 k} \mathrm{Sq}^{2} \mathrm{Sq}^{1} i\right\}$. Letting $N^{r+4 k+3}$ be a Spin manifold with $w \in H^{4 k+3}\left(N ; Z_{2}\right)$ to realize a class in $\tilde{\Omega}_{r+4 k+3}^{\text {Spin }}\left(K\left(Z_{2}, r\right)\right) \cong H_{4 k+3}\left(B \operatorname{Spin} ; Z_{2}\right)$, the value of $U\left\{\mathrm{Sq}^{4 k} \mathrm{Sq}^{2} \mathrm{Sq}^{1} i\right\}$ on $(N, w)$ is

$$
\begin{aligned}
\mathrm{Sq}^{4 k} \mathrm{Sq}^{2} \mathrm{Sq}^{1} w[N] & =v_{4 k} \mathrm{Sq}^{2} \mathrm{Sq}^{1} w[N]=\left\{v_{2} v_{4 k} \mathrm{Sq}^{1} w+\mathrm{Sq}^{2} v_{4 k} \mathrm{Sq}^{1} w\right\}[N] \\
& =\left\{v_{1} \mathrm{Sq}^{2} v_{4 k} w+\mathrm{Sq}^{1} \mathrm{Sq}^{2} v_{4 k} \cdot w\right\}[N]=\left\{\mathrm{Sq}^{3} v_{4 k} \cdot w\right\}[N] .
\end{aligned}
$$

Thus, the only class in $H^{4 k+3}\left(B \operatorname{Spin} ; Z_{2}\right)$ which can vanish on the image of $g$ is $\mathrm{Sq}^{3} v_{4 k}$. Thus $\mathrm{Sq}^{1} \theta=\mathrm{Sq}^{3} v_{4 k}=\mathrm{Sq}^{1} \mathrm{Sq}^{2} v_{4 k}$ and $\theta=\mathrm{Sq}^{2} v_{4 k}$.

Finally, for the case $k=2$, one could presumably redo all of the calculations of the previous section for the case $k=1$. However, being given $M^{18}$ and a class $z \in H^{8}(M ; Z)$ with Wu class $v(M)=1+v_{4}^{\prime}+v_{8}^{\prime}$ one can let $u \in H^{4}\left(H P^{2} ; Z\right)$ and consider $u \otimes z \in H^{12}\left(H P^{2} \times M ; Z\right)$ so that

$$
\begin{aligned}
\rho z \mathrm{Sq}^{2} \rho z[M] & =\rho(u \otimes z) \mathrm{Sq}^{2} \rho(u \otimes z)\left[H P^{2} \times M\right] \\
& =\rho(u \otimes z) \mathrm{Sq}^{2} v_{12}\left[H P^{2} \times M\right] \\
& =\rho(u \otimes z) \mathrm{Sq}^{2}\left(\rho u \otimes v_{8}^{\prime}\right)\left[H P^{2} \times M\right] \\
& =\rho z \mathrm{Sq}^{2} v_{8}^{\prime}[M] .
\end{aligned}
$$

Thus, the result for $k=3$ implies it for $k=2$. 
COROllary $5.2[\mathbf{W}] . \mathrm{Sq}^{3} v_{4 k}=1 \mathrm{Sq}^{4 k} \mathrm{Sq}^{2} \mathrm{Sq}^{1}$ is zero in every closed Spin manifold of dimension $8 k+2$.

Proof. Having seen that $\theta=\mathrm{Sq}^{2} v_{4 k}$ gives this.

Note. With the exception of the case $k=2$, one has shown that this is the only nonzero class of dimension $4 k+3$ which is zero in every manifold of dimension $8 k+2($ or $8 k-1)$.

COROLlaRY 5.3. For a closed spin manifold $M^{8 k+2}$ of dimension $8 k+2$, $w_{4} w_{8 k-2}[M]=v_{4 k} \mathrm{Sq}^{2} v_{4 k}[M]$ is the rank modulo 2 of the form [, ] on integral cohomology.

Proof. Consider the form

$$
[,]: H^{4 k}(M ; Z) \otimes H^{4 k}(M ; Z) \rightarrow Z_{2}:[x, y]=\rho x \mathrm{Sq}^{2} \rho y[M] .
$$

By standard facts about forms (as in [LMP, §2]), there is a class $v \in H^{4 k}(M ; Z)$, well-defined modulo the annihilator of the form, for which $[x, y]=[x, x]$ for all $x$ and $[v, v]$ is the rank modulo 2 of the form [ , ]. In $H^{*}\left(B \operatorname{Spin} ; Z_{2}\right)$, it is well known [ABP] that $\mathrm{Sq}^{1} v_{4 k}=0$, and the kernel of $\mathrm{Sq}^{1}$ is the image of the reduction of $H^{*}(B \operatorname{Spin} ; Z)$. Thus there is a class $w \in H^{*}(B \operatorname{Spin} ; Z)$ with $\rho w=v_{4 k}$. By the proposition $\tau^{*}(w) \in H^{4 k}(M ; Z)$ is a suitable choice for $v$ and so the rank mod 2 of $\left[\right.$, ] is $\left[\tau^{*}(w), \tau(w)\right]=\rho \tau^{*}(w) \mathrm{Sq}^{2} \rho \tau^{*}(w)[M]=v_{4 k} \mathrm{Sq}^{2} v_{4 k}[M]$. Finally,

$$
\begin{aligned}
v_{4 k} \mathrm{Sq}^{2} v_{4 k}[M] & =\mathrm{Sq}^{4 k} \mathrm{Sq}^{2} v_{4 k}[M] \\
& =\left\{\mathrm{Sq}^{4} \mathrm{Sq}^{4 k-2} v_{4 k}+\left(\begin{array}{c}
4 k-3 \\
4
\end{array}\right) \mathrm{Sq}^{4 k+2} v_{4 k}\right\}[M] \\
& =v_{4} \mathrm{Sq}^{4 k-2} v_{4 k}[M]
\end{aligned}
$$

and since $v_{i}(M)=0$ for $i \not \equiv 0(4), v_{4}=w_{4}$ and $\mathrm{Sq}^{4 k-2} v_{4 k}=w_{8 k-2}$ for $w=\mathrm{Sq} v$.

ObSERVATION. There is no class $y \in H^{4 k+2}\left(B \operatorname{Spin} ; Z_{2}\right)$ with $k>0$ so that for all closed Spin manifolds $M^{8 k+2}$ and $x \in H^{4 k}\left(M ; Z_{2}\right)$ one has

$$
x \mathrm{Sq}^{2} x[M]=x \tau^{*}(y)[M] .
$$

PROOF. From the calculations in the previous section (valid for $k \geqslant 1$ ) one has a class $a \in \pi_{r+4 k+3}\left(M \operatorname{Spin} \wedge Y_{r}\right)$ for which $U \delta \sigma i \mathrm{Sq}^{2} i$ has a nonzero value. In the sequence

$$
\pi_{r+4 k+3}\left(M \operatorname{Spin} \wedge Y_{r}\right) \stackrel{\partial}{\rightarrow} \tilde{\Omega}_{8 k+2}^{\text {Spin }}\left(K\left(Z_{2}, 4 k\right)\right) \rightarrow H_{4 k+2}\left(B \text { Spin } ; Z_{2}\right)
$$

$\partial a$ is given by an $M^{8 k+2}$ and class $x$ with $x \mathrm{Sq}^{2} x[M] \neq 0$ and so that $x \tau^{*}(y)[M]$ $=0$ for all $y$.

Note. This shows that the restriction to integral classes was absolutely crucial.

ObSeRvation. There is no class $y \in H^{4 k+4}\left(B\right.$ Spin; $\left.Z_{2}\right)$ so that for all closed Spin manifolds $M^{8 k+6}$ and $z \in H^{4 k+2}(M, Z)$ one has

$$
\rho z \mathrm{Sq}^{2} \rho z[M]=\rho z \tau^{*}(y)[M] .
$$


Proof. Let $M^{8 k+6}=H P^{2 k} \times \mathbf{C} P^{3}$ and $z=u^{k} a$ where $u \in H^{4}\left(H P^{2 k} ; Z\right), a \in$ $H^{2}\left(\mathbf{C} P^{3} ; Z\right)$. Then $\rho z \mathrm{Sq}^{2} \rho z[M]=\rho\left(u^{k} a\right) \rho\left(u^{k} a^{2}\right)[M] \neq 0$. Also $w(M)=$ $(1+\rho u)^{2 k+1}(1+\rho a)^{4}=(1+\rho u)^{2 k+1}$ and for any $y \in H^{4 k+4}\left(B \operatorname{Spin} ; Z_{2}\right), \tau^{*}(y)$ $=\lambda \rho u^{k+1}$ for some $\lambda \in Z_{2}$. Thus $\rho z \tau^{*}(y)[M]=\lambda \rho u^{2 k+1} \rho a[M]=0$.

OBSERVATION. There is no class $y \in H^{4 k+1}\left(B \operatorname{Spin} ; Z_{2}\right)$ with $k>0$ so that for all closed Spin manifolds $M^{8 k}$ and $z \in H^{4 k-1}(M ; Z)$ one has

$$
\rho z \mathrm{Sq}^{2} \rho z[M]=\rho z \tau *(y)[M] .
$$

Proof. Let $M^{8 k}=H P^{2 k-2} \times G_{2}\left(R^{6}\right)$, where $G_{2}\left(R^{6}\right)$ is the Grassmannian of 2-planes in $R^{6}$. Then $H^{*}\left(G_{2}\left(R^{6}\right) ; Z_{2}\right)$ is the $Z_{2}$ polynomial ring on the universal Stiefel-Whitney classes $w_{1}, w_{2}$ modulo the relations $\left(1 /\left(1+w_{1}+w_{2}\right)\right)_{i}=0$ if $i>4$. One has $w\left(G_{2}\left(R^{6}\right)\right)=\left(1+w_{1}+w_{2}\right) 6 /\left(1+w_{1}^{2}\right)$, so that $G_{2}\left(R^{6}\right)$ is a Spin manifold, and for any $y \in H^{4 k+1}\left(B \operatorname{Spin} ; Z_{2}\right), \tau^{*}(y)=0$ in $M$ since all odd dimensional Stiefel-Whitney classes are zero. Let $a=\beta w_{2} \in H^{3}\left(G_{2}\left(R^{6}\right) ; Z\right)$ be the integral Bockstein of $w_{2}$, so $\rho a=\rho \beta w_{2}=\mathrm{Sq}^{1} w_{2}=w_{1} w_{2}$, and let $z$ be $u^{k-1} a$. Then

$$
\rho z \mathrm{Sq}^{2} \rho z[M]=\mathrm{Sq}^{1} w_{2} \mathrm{Sq}^{2} \mathrm{Sq}^{1} w_{2}\left[G_{2}\left(R^{6}\right)\right] \neq 0 .
$$

In dimensions $8 k+4$ with $k>0$, one may similarly consider $H P^{2 k-2} \times M^{12}$ where $M^{12}$ is a Spin manifold having a class $a \in H^{5}(M ; Z)$ with $\rho a \mathrm{Sq}^{2} \rho a[M] \neq 0$, and may let $z=u^{k-1} a$ to give $\rho z \mathrm{Sq}^{2} \rho z\left[H P^{2 k-2} \times M\right] \neq 0$. The Wu class of $M$ has the form $1+v_{4}\left(v_{i}=0\right.$ if $i \not \equiv 0 \bmod 4$ or $\left.i>6\right)$ so $w(M)=1+w_{4}+w_{6}+w_{7}+w_{8}$ and by Wilson $[\mathbf{W}], w_{7}=0$. Thus $w\left(H P^{2 k-2} \times M\right)$ consists entirely of even dimensional classes, and for any $y \in H^{4 k+3}\left(B \operatorname{Spin} ; Z_{2}\right), \tau^{*}(y)=0$.

By calculation, one can show that $\left(M^{12}, a\right)$ exists. To exhibit such calculations would be a travesty; one would prefer a specific example.

Note. In dimensions $8 k$ and $8 k+4$, with $k=0, y=0$ would give the universal class. Similarly, $y=0$ suffices for $\bmod 2$ cohomology in dimensions $8 k+2$ with $k=0$.

6. A technical extension. Having seen that the main result does not hold for arbitrary mod 2 cohomology classes, one is led to ask whether weaker conditions than being reduced integral are sufficient. One does, in fact, have

Proposition 6.1. For a closed Spin manifold $M^{8 k+2}$ of dimension $8 k+2$ and class $x \in H^{4 k}\left(M ; Z_{2}\right)$, one has

$$
x \mathrm{Sq}^{2} x[M]=x \mathrm{Sq}^{2} v_{4 k}[M]
$$

if $\mathrm{Sq}^{1} x=0$, i.e. if $x$ is the reduction of a $Z_{4}$ class.

Corollary 6.2. For a closed Spin manifold $M^{8 k+2}$ of dimension $8 k+2$, $w_{4} w_{8 k-2}[M]$ is the rank modulo 2 of the form $[$,$] on \left(\mathrm{ker} \mathrm{Sq}^{1}\right)^{4 k}$ or $H^{4 k}\left(M: Z_{2} s\right)$ for any $s>1$. 
Note. The results of $[\mathbf{L M P}]$ relate the form $(x, y)=x \mathrm{Sq}^{1} y[M]$ to the torsion in homology in a very precise way. These results indicate that there is some relation on the torsion for Spin manifolds of dimension $8 k+2$ because the rank of the form is independent of $s$, but the relation is vague.

Proof. One has a cofibration

$$
\sum^{r-4 k} K\left(Z_{4}, 4 k\right) \rightarrow K\left(Z_{4}, r\right) \rightarrow W_{r}
$$

giving an exact sequence

$$
\pi_{r+4 k+3}\left(M \operatorname{Spin} \wedge W_{r}\right) \stackrel{\partial}{\rightarrow} \underset{\Omega}{\tilde{\Omega}_{8 k+2}} \operatorname{Spin}\left(K\left(Z_{4}, 4 k\right)\right) \stackrel{a}{\rightarrow} H_{4 k+2}\left(B \operatorname{Spin} ; Z_{4}\right) \rightarrow \cdots .
$$

One may then analyze $\tilde{H}^{*}\left(W_{r} ; Z_{2}\right)$ and find

$$
\begin{array}{ll}
\operatorname{dim}(r+4 k+1) & {\left[\mathrm{Sq}^{4 k+1} i_{r}\right],} \\
\operatorname{dim}(r+4 k+2) & {\left[\mathrm{Sq}^{4 k+2} i_{r}\right], \delta \sigma^{r-4 k} i_{4 k} \beta i_{4 k},} \\
\operatorname{dim}(r+4 k+3) & {\left[\mathrm{Sq}^{4 k+3} i_{r}\right],\left[\mathrm{Sq}^{4 k+2} \beta i_{r}\right], \delta \sigma^{r-4 k} i_{4 k} \mathrm{Sq}^{2} i_{4 k},}
\end{array}
$$

where $\beta$ denotes the Bockstein. Since $\mathrm{Sq}^{2}\left[\mathrm{Sq}^{4 k+1} i_{r}\right]$ goes to $\mathrm{Sq}^{4 k+2} \mathrm{Sq}^{1} i_{r}=0$ in $K\left(Z_{4}, r\right)$, one has $\mathrm{Sq}^{2}\left[\mathrm{Sq}^{4 k+1} i_{r}\right]=\mu \delta \sigma^{r-4 k} i_{4 k} \mathrm{Sq}^{2} i_{4 k}$ for some $\mu \in Z_{2}$.

If one considers the maps $K(Z, n) \rightarrow K\left(Z_{4}, n\right)$, one has an induced map $X_{r} \stackrel{b}{\rightarrow} W_{r}$ so that $b^{*}: \tilde{H}^{*}\left(W_{r} ; Z_{2}\right) \rightarrow \tilde{H}^{*}\left(X_{r} ; Z_{2}\right)$ sends $\left[\mathrm{Sq}^{4 k+1} i_{r}\right]$ to $\left[\mathrm{Sq}^{4 k+1} i_{r}\right]$. Thus $\mathrm{Sq}^{2}\left[\mathrm{Sq}^{4 k+1} i_{r}\right] \neq 0$ in $W_{r}$, because its image in $X_{r}$ is nonzero, and $\mu \neq 0$.

Thus

$$
\phi: \tilde{\Omega}_{8 k+2}^{\mathrm{Spin}}\left(K\left(Z_{4}, 4 k\right)\right) \rightarrow Z_{2}:(M, f) \rightarrow\left(f^{*} i\right) \mathrm{Sq}^{2} f^{*} i[M]
$$

is zero on the image of $\partial$, and is given by a homomorphism $\operatorname{im} a \rightarrow Z_{2}$. Since all torsion in $H_{*}(B$ Spin; $Z)$ is of order $2, H_{4 k+2}\left(B \operatorname{Spin} ; Z_{4}\right) \stackrel{\rho}{\rightarrow} H_{4 k+2}\left(B \operatorname{Spin} ; Z_{2}\right)$ is monic, and there is a class $\theta \in H^{4 k+2}\left(B \operatorname{Spin} ; Z_{2}\right)$ so that

$$
x \mathrm{Sq}^{2} x[M]=\tau^{*}(\theta) \cdot x[M]
$$

for all closed Spin $M^{8 k+2}$ and $x \in(\operatorname{ker~Sq})^{4 k}=\rho H^{4 k}\left(M ; Z_{4}\right)$.

One must again identify $\theta$, but this is just a repetition of the arguments. $\theta$ is well defined only modulo the image of $\mathrm{Sq}^{1}$, hence is determined by $\mathrm{Sq}^{1} \boldsymbol{\theta}$, and $\mathrm{Sq}^{1} \theta$ is zero in all Spin $M^{8 k+2}$. By uniqueness, $\theta=\mathrm{Sq}^{2} v_{4 k}$ mod image $\mathrm{Sq}^{1}$ for $k \geqslant 3$, and this implies that $\theta$ can be taken to be $\mathrm{Sq}^{2} v_{4 k}$ for smaller $k$.

Note. The argument for $Z_{4}$ is really identical with that for $Z$ classes, and this presentation has simply used the $Z$ argument to give the Steenrod operations in $W_{r}$. The equivalence of the ranks of the forms for $Z$ and $Z_{2} s$ cohomology follows from the fact that the class $v_{4 k}$ is reduced integral.

Note. One can analyze the form [, ] simply by knowing $H^{*}\left(M ; Z_{2}\right)$ as algebra over the Steenrod algebra, since that gives $\left.(\operatorname{kerSq})^{1}\right)^{4 k}$. Working with $\rho H^{4 k}(M ; Z)$ would require extra information. 
Comment. This extension to $Z_{4}$ classes was inspired by a suggestion of Steven $M$. Kahn. Using this extension the methods of $[\mathbf{K}]$ may be applied to prove

Proposition 6.3. If $M^{8 k+2}$ is a closed Spin manifold of dimension $8 k+2$ with an involution $T$ of odd type preserving the Spin structure, then

$$
w_{4} w_{8 k-2}[M] \equiv \chi\left(F^{8^{*}}\right) \equiv \chi\left(F^{8^{*}+4}\right) \quad(\bmod 2)
$$

where $\chi$ is the Euler characteristic and $F^{8^{*+j}}$ is the part of the fixed set of $T$ having dimension $j \bmod 8$.

\section{REFERENCES}

[ABP] D. W. Anderson, E. H. Brown, Jr., and F. P. Peterson, The structure of the Spin cobordism ring, Ann. of Math. (2) 86 (1967), 271-298.

[B1] W. Browder, Remark on the Poincaré duality theorem, Proc. Amer. Math. Soc. (2) 13 (1962), $927-930$.

[B2] W. Browder, Torsion in H-spaces, Ann. of Math. (2) 74 (1961), 24-51.

[B3] E. H. Brown, Jr., Generalizations of the Kervaire invariant, Ann. of Math. (2) 95 (1972), 368-383.

[F] E. E. Floyd, The number of cells in a nonbounding manifold, Ann. of Math. (2) 98 (1973), 210-225.

[K] S. M. Kahn, Involutions on odd-dimensional manifolds and the de Rham invariant, Topology (to appear).

[LMP] G. Lusztig, J. Milnor, and F. P. Peterson, Semicharacteristics and cobordism, Topology 8 (1969), 357-359.

[S] R. E. Stong, Appendix: Calculation of $\Omega_{1}^{\text {Spin }}(K(Z, 4))$, Unified String Theories, edited by M. Green and D. Gross, World Scientific Press, Singapore, 1986, pp. 430-437.

[W] W. S. Wilson, A new relation on the Stiefel-Whitney classes of spin manifolds, Illinois J. Math. 17 (1973), 115-127.

Department of Mathematics, Rutgers University, New Brunswick, New Jersey 08903

Deparment of Mathematics, University of Virginia, Charlottesville, Virginia 22903 